\title{
Avoiding patterns in irreducible permutations
}

\author{
Jean-Luc Baril|k
}

LE2I UMR-CNRS 6306, Université de Bourgogne, Dijon, France

received $17^{\text {th }}$ Jan. 2013, revised $18^{\text {th }}$ Dec. 2014, accepted $3^{\text {rd }}$ Dec. 2015.

\begin{abstract}
We explore the classical pattern avoidance question in the case of irreducible permutations, i.e., those in which there is no index $i$ such that $\sigma(i+1)-\sigma(i)=1$. The problem is addressed completely in the case of avoiding one or two patterns of length three, and several well known sequences are encountered in the process, such as Catalan, Motzkin, Fibonacci, Tribonacci, Padovan and Binary numbers. Also, we present constructive bijections between the set of Motzkin paths of length $n-1$ and the sets of irreducible permutations of length $n$ (respectively fixed point free irreducible involutions of length $2 n$ ) avoiding a pattern $\alpha$ for $\alpha \in\{132,213,321\}$. This induces two new bijections between the set of Dyck paths and some restricted sets of permutations.
\end{abstract}

Keywords: Pattern avoiding permutation; irreducible permutation; succession; involution; Motzkin path

\section{Introduction and notation}

Let $\mathcal{S}_{n}$ be the set of permutations on $[n]=\{1,2, \ldots, n\}$, i.e., all one-to-one correspondences from $[n]$ into itself. Let $\mathcal{S}$ be the set of all permutations. We represent a permutation $\sigma \in \mathcal{S}_{n}$ in one-line notation $\sigma=\sigma_{1} \sigma_{2} \cdots \sigma_{n}$ where $\sigma_{i}=\sigma(i)$ for all $i \in[n]$. We denote by $\sigma^{r}, \sigma^{c}$ and $\sigma^{-1}$ the classical symmetries of $\sigma$, i.e., the reverse $\sigma^{r}=\sigma_{n} \cdots \sigma_{1}$, the complement $\sigma^{c}=\left(n-\sigma_{1}+1\right) \cdots\left(n-\sigma_{n}+1\right)$ and the inverse.

Let $q=q_{1} \cdots q_{k}, k \geq 1$, be a sequence of pairwise different positive integers. The reduction $\operatorname{red}(q)$ of $q$ is the permutation in $S_{k}$ obtained from $q$ by replacing the $i$-th smallest number of $q$ with $i$ for $1 \leq i \leq k$. For instance, if $q=53841$ then we have $\operatorname{red}(q)=42531$. A permutation $\sigma \in \mathcal{S}_{n}$ avoids the pattern $\pi \in \mathcal{S}_{k}, k \geq 1$, if and only if there does not exist a sequence of indices $1 \leq i_{1}<i_{2}<\cdots<i_{k} \leq n$ such that $\sigma_{i_{1}} \sigma_{i_{2}} \cdots \sigma_{i_{k}}$ is order-isomorphic to $\pi$ (see [15, 20]), i.e. such that $\pi=\operatorname{red}\left(\sigma_{i_{1}} \sigma_{i_{2}} \cdots \sigma_{i_{k}}\right)$. We denote by $\mathcal{S}_{n}(\pi)$ the set of permutations in $\mathcal{S}_{n}$ avoiding the pattern $\pi$. For example, if $\pi=123$ then $52143 \in \mathcal{S}_{5}(\pi)$ while $\mathbf{2 1 5 3 4} \notin \mathcal{S}_{5}(\pi)$. In the case where $\sigma$ does not avoid $\pi$ (or equivalently contains $\pi$ ), $\pi$ is said to be involved in $\sigma$ which is denoted $\pi \preceq \sigma$. A set $\mathcal{F}$ of permutations is called a permutation class if it is closed downwards under this involvement relation. Many classical sequences in combinatorics appear as the cardinality of pattern-avoiding permutation classes. A large number of these results were firstly obtained by West and Knuth [8, 15, 20, 21, 22] (see the surveys of Kitaev and Mansour [7, 10]). Also Bóna [3] has written a book that is dedicated to the notion of pattern avoiding permutations. Some generalizations of pattern avoidance can be viewed in [2, 12].

*email: $\{$ barjl\}@u-bourgogne.fr

1365-8050 (c) 2016 Discrete Mathematics and Theoretical Computer Science (DMTCS), Nancy, France 
A succession in a permutation $\sigma \in \mathcal{S}_{n}$ is a pair $\left(\sigma_{i}, \sigma_{i+1}\right), 1 \leq i \leq n-1$, with $\sigma_{i+1}-\sigma_{i}=1$. For instance, the pair $(3,4)$ is the only one succession in $\sigma=53421$. Using the terminology of Atkinson and Stitt (see [1]), a permutation with no successions will be also called irreducible. They show how the notion of irreducibility plays, in some sense, a dual role compared to indecomposability (a permutation $\sigma=\sigma_{1} \cdots \sigma_{n}$ is indecomposable if there does not exist $p \leq n-1$ such that $\sigma_{1} \cdots \sigma_{p}$ is a permutation of $[p]$ ). Indeed, irreducible permutations are crucial for the construction of a basis of some wreath products $\mathcal{C} \prec \mathcal{I}$ where $\mathcal{C}$ is a class of permutations and $\mathcal{I}$ is the class of identity permutations (see [1]). On the other hand, an irreducible permutation can be also viewed as a permutation avoiding the bi-vincular pattern $(12,\{1\},\{1\})$ (see [11]).

Let $\mathcal{S}_{n}^{\text {irr }}$ be the set of irreducible permutations of length $n$. For example, we have $\mathcal{S}_{3}^{\text {irr }}=\{132,213,321\}$. The cardinality of the sets $\mathcal{S}_{n}^{i r r}$ is known (see [14, 18, 19]) and given by

$$
(n-1) ! \sum_{k=0}^{n-1}(-1)^{k} \frac{n-k}{k !} .
$$

More generally, permutations with a given number of successions also appeared in combinatorial theory literature (see [6, 13]).

In this paper, we explore the classical pattern avoidance question in the case of irreducible permutations. The problem is addressed completely in the case of avoiding one or two patterns of length three, and several well-known sequences are encountered, such as the Catalan, Motzkin, Fibonacci, Binary, Tribonacci and Padovan numbers. In Section 2, we present general enumerative results for sets of permutations that are expanded (i.e., closed under inflation) and closed under deflation. In particular, we give bivariate generating functions according to the length of permutations and the number of successions. Generalizing the two concepts of inflation and deflation for sets of fixed point free involutions, we obtain similar results for involutions. In Section 3, we give exhaustive enumerative results for sets of irreducible permutations avoiding one pattern of length three. For the sets counted by the Motzkin numbers, we exhibit bijections between them and the set of Motzkin paths. In Section 4, we focus on sets of irreducible permutations avoiding two patterns of length three. In Sections 5 and 6, we study irreducible (fixed point free) involutions avoiding one pattern of length three. Exhaustive enumerative results are obtained, and we construct bijections between fixed point free irreducible involutions of length $2 n$ avoiding $\alpha \in\{132,213,321\}$ and Motzkin paths of length $n-1$.

\section{Preliminaries}

A set $\mathcal{F}$ of permutations is said to be closed under inflation (also called expanded, [1]) if whenever a permutation $\sigma=\beta i \gamma \in \mathcal{F}$ so is the permutation obtained by replacing $i$ with $i(i+1)$, after increasing by one each other's values greater than $i$ in $\sigma$. A set $\mathcal{F}$ of permutations is said to be closed under deflation if whenever a permutation $\sigma=\beta i(i+1) \gamma$ so is the permutation obtained by replacing $i(i+1)$ with $i$, after decreasing by one each other's values greater than $(i+1)$ in $\sigma$. In the following, these two last elementary transformations will be respectively called inflation and deflation of $\sigma$. Obviously, a set $\mathcal{F}$ of irreducible permutations is closed under deflation since its elements do not contain any successions. A set closed under pattern involvement is closed under deflation. Moreover, a set closed under pattern involvement is expanded if and only if every basis element is irreducible (see Lemma 5, [1]).

In this section we provide general lemmas about generating functions for sets of permutations and fixed point free involutions which are expanded and closed under deflation. 
Lemma 1 Let $\mathcal{F}$ be a set of permutations which is expanded and closed under deflation; let $\mathcal{G}$ be the set of its irreducible permutations. We denote by $g(x)$ the generating function of $\mathcal{G}$, and $f(x, y)$ the bivariate generating function of $\mathcal{F}$ where the coefficient of $x^{n} y^{k}$ is the number of permutations of length $n$ with exactly $k$ successions. Then we have

$$
f(x, y)=g\left(\frac{x}{1-y x}\right) .
$$

In particular, the generating function of $\mathcal{F}$ is $g(x /(1-x))$.

Proof: Let $\mathcal{F}_{n}^{k}, n \geq 1,0 \leq k \leq n-1$, be the set of permutations of length $n$ in $\mathcal{F}$ containing exactly $k$ successions. Obviously, we have $\mathcal{F}_{n}^{i r r}=\mathcal{G}_{n}=\mathcal{F}_{n}^{0}$ for $n \geq 1$. Let $\sigma$ be a permutation in $\mathcal{F}_{n}^{k}$, i.e, $\sigma$ contains exactly $k$ successions. We define the succession set $I_{\sigma}$ of indices $i \in[n-1]$ such that $\left(\sigma_{i}, \sigma_{i+1}\right)$ is a succession of $\sigma$, and $J_{\sigma}=\left\{i \in[n], i-1 \in I_{\sigma}\right\}$. Since $\mathcal{F}$ is expanded and closed under deflation, a permutation $\pi \in \mathcal{F}_{n-\left|I_{\sigma}\right|}^{i r r}$ can be uniquely obtained from a permutation $\sigma \in \mathcal{F}_{n}^{k}$ by deleting all entries $\sigma_{i}$, $i \in J_{\sigma}$ and reducing the result to a permutation of $\mathcal{F}_{n-\left|I_{\sigma}\right|}^{i r r}$. This construction produces a simple bijection from $\mathcal{F}_{n-\left|I_{\sigma}\right|}^{i r r}$ to the set $\mathcal{F}_{n}^{I_{\sigma}}$ of permutations in $\mathcal{F}_{n}$ having the succession set equal to $I_{\sigma}$.

Since inflations of terms in an irreducible permutation correspond exactly to the terms in the geometric series expansion $\frac{x}{1-y x}=x+y x^{2}+y^{2} x^{3}+\ldots$, the above bijection implies

$$
f(x, y)=g\left(x+y x^{2}+y^{2} x^{3}+\ldots\right)=g\left(\frac{x}{1-y x}\right) .
$$

Now we give a similar Lemma for fixed point free involutions. In this case, we slightly modify the two concepts of inflation and deflation. Let $\mathcal{F}$ be a set of involutions with no fixed points. We say that $\mathcal{F}$ is expanded (closed under inflation) if whenever an involution $\sigma \in \mathcal{F}$ and $(i, j), 1 \leq i<j \leq n$, such that $\sigma_{i}=j$, so is the permutation $\sigma^{\prime}$ obtained from $\sigma$ by replacing $\sigma_{i}$ with $\left(\sigma_{i}+1\right)\left(\sigma_{i}+2\right), \sigma_{j}$ with $\sigma_{j}\left(\sigma_{j}+1\right)$, and increasing by inc $(k)=\operatorname{card}\{\ell \in\{i+1, j+1\}, \ell \leq k\}$ each other's values $k$. For instance, if $\sigma=34127856, i=2, j=4$, then $\sigma^{\prime}=4561239$ (10) 78 . On the other hand, we say that $\mathcal{F}$ is closed under deflation if whenever an involution $\sigma \in \mathcal{F}$ and $(i, j), 1 \leq i<j \leq n-1$, such that $\sigma_{i}=j, \sigma_{i+1}=j+1$, so is the permutation $\sigma^{\prime}$ obtained from $\sigma$ by deleting $(i+1)$ and $(j+1)$ and reducing the result to a permutation. Obviously the set of all fixed point free involutions is expanded and closed under deflation.

Lemma 2 Let $\mathcal{F}$ be a set of fixed point free involutions which is expanded and closed under deflation; let $\mathcal{G}$ be the set of its irreducible involutions. We denote by $g(x)$ the generating function of $\mathcal{G}$, and $f(x, y)$ the bivariate generating function of $\mathcal{F}$ where the coefficient of $x^{n} y^{k}$ is the number of involutions of length $n$ with exactly $k$ successions. Then we have

$$
f(x, y)=g\left(\sqrt{\frac{x^{2}}{1-y^{2} x^{2}}}\right) .
$$

In particular, the generating function of $\mathcal{F}$ is $g\left(\sqrt{x^{2} /\left(1-x^{2}\right)}\right)$. 
Proof: This proof is a simple counterpart of the previous one. It suffices to remark that an involution with no fixed points is necessarily of even length.

Lemma 1 ensures that if we know the generating function of a set $\mathcal{G}$ of irreducible permutations, then we can easily obtain the bivariate generating function (according to the length and the number of successions) of the wreath product $\mathcal{G} \backslash \mathcal{I}$ where $\mathcal{I}$ is the class of identity permutations (the wreath product $\mathcal{G} \backslash \mathcal{I}$ is the closure under inflation of $\mathcal{G}$ ). An immediate consequence is: if $\mathcal{G}$ is a set of irreducible permutations such that the wreath product $\mathcal{G} \geq \mathcal{I}$ is enumerated by the Catalan numbers ([16], A00018) then $\mathcal{G}$ is enumerated by the Motzkin numbers ([16], A001006). Therefore, irreducible permutations of $\mathcal{S}_{n}(\alpha)$, $n \geq 1, \alpha \in\{321,213,132\}$, are enumerated by the Motzkin numbers (see Theorem 2).

\section{Avoiding a pattern of length 3}

In this section, we study the avoidance of one pattern of length 3 for irreducible permutations. The first part uses arguments with generating functions while the second part presents bijective proofs for the sets of permutations counted by Motzkin numbers. All enumerative results of this section are listed in Table 1.

\subsection{Using generating functions}

For $\alpha=231$, Atkinson and Stitt give a proof of Remark 1 (see [1], Section 6.1). The bijection $\chi: \sigma \rightarrow$ $\left(\sigma^{c}\right)^{r}$, allows to conclude for $\alpha=312$.

Remark 1 For $\alpha \in\{231,312\}$, the generating function for the sets $\mathcal{S}_{n}^{\text {irr }}(\alpha), n \geq 0$, of irreducible permutations avoiding $\alpha$ is given by

$$
-1+\frac{2(1+x)}{1+x+x^{2}+\sqrt{\left(1-x+x^{2}\right)^{2}-8 x^{2}}}
$$

(see [16], A078481).

Theorem 1 The generating function for the sets $\mathcal{S}_{n}^{\text {irr }}(123), n \geq 0$, of irreducible permutations of length $n$ avoiding the pattern 123 is given by (see [16], A114487):

$$
\frac{2}{1+2 x^{2}+\sqrt{1-4 x}} \text {. }
$$

Proof: Let $\sigma$ be a permutation in $\mathcal{S}_{n}(123) \backslash \mathcal{S}_{n}^{\text {irr }}(123)$. It necessarily contains a succession $(k, k+1)$, $1 \leq k \leq n-1$; let us take the leftmost succession. Then $\sigma$ can be written $\sigma=u k(k+1) v$ where $1 \leq k \leq n-1$, such that $u$ and $v$ are two subsequences of $[n]$. Since $\sigma$ avoids 123 , the set of values in $u$ necessarily equals to the interval $[k+2, n]$. So $v$ belongs to $\mathcal{S}_{k-1}(123), u$ avoids the pattern 123 and does not contain any successions. Let $f(x)$ be the generating function for the sets $\mathcal{S}_{n}^{\text {irr }}(123), n \geq 0$, the above decomposition of $\sigma$ implies

$$
c(x)-f(x)=x^{2} \cdot f(x) \cdot c(x),
$$

where $c(x)=\frac{1-\sqrt{1-4 x}}{2 x}$ is the generating function for the sets $\mathcal{S}_{n}(123)$, i.e., the generating function for the well-known Catalan numbers (see [16], A00018). We deduce:

$$
f(x)=\frac{c(x)}{1+x^{2} \cdot c(x)}=\frac{2}{1+2 x^{2}+\sqrt{1-4 x}} .
$$


Theorem 2 For $\alpha \in\{321,213,132\}$, the sets $\mathcal{S}_{n}^{\text {irr }}(\alpha), n \geq 1$, are enumerated by the Motzkin numbers (see [16], A001006).

Proof: For $\alpha \in\{321,213,132\}$, the set $\mathcal{S}(\alpha)=\bigcup_{n=1}^{\infty} \mathcal{S}_{n}(\alpha)$ is expanded and closed under deflation. Let $g(x)$ be the generating function of $\mathcal{S}^{i r r}(\alpha)$; Lemma 1 of Section 2 proves that $c(x)=g(x /(1-x))$ and thus:

$$
g(x)=c(x /(1+x)) .
$$

More precisely, the set $\mathcal{S}_{n}^{i r r}(\alpha), n \geq 1$, is enumerated by the $(n-1)$-th term of the Motzkin sequence ([16], A001006).

In fact, Lemma 1 yields the more general result:

Theorem 3 For $\alpha \in\{321,213,132\}$, let $\mathcal{S}(\alpha)$ be the set of permutations avoiding $\alpha$, and $f(x, y)$ its bivariate generating function, where the coefficient of $x^{n} y^{k}$ is the number of permutations of length $n$ with $k$ successions in $\mathcal{S}(\alpha)$. Then we have

$$
f(x, y)=c\left(\frac{x}{1+(1-y) x}\right),
$$

where $c(x)$ is the generating function for the Catalan numbers.

\begin{tabular}{|c|c|c|c|}
\hline Pattern & Sequence & Sloane & $a_{n}, n \geq 1$ \\
\hline$\{231\},\{312\}[1]$ & $-1+\frac{2(1+x)}{1+x+x^{2}+\sqrt{\left(1-x+x^{2}\right)^{2}-8 x^{2}}}$ & $A 078481$ & $1,1,3,7,19,53,153$ \\
\hline$\{123\}$ & $\frac{2}{1+2 x^{2}+\sqrt{1-4 x}}$ & $A 114487$ & $1,1,3,10,31,98,321$ \\
\hline$\{321\},\{213\},\{132\}$ & $\frac{1-x-\sqrt{1-2 x-3 x^{2}}}{2 x^{2}}$ & $A 001006$ & $1,1,2,4,9,21,51$ \\
\hline
\end{tabular}

Tab. 1: Wilf-equivalence classes for patterns of length 3 in irreducible permutations.

\subsection{Bijective proofs}

A Motzkin path of length $n$ is a lattice path starting at $(0,0)$, ending at $(n, 0)$, and never going below the $x$-axis, consisting of up steps $U=(1,1)$, horizontal steps $H=(1,0)$, and down steps $D=(1,-1)$. A Dyck path of length $2 n$ is a Motzkin path of the same length that does not contain any horizontal steps. Dyck paths of length $2 n$ are enumerated by the well-known Catalan numbers ([16], A00018) and Motzkin paths by the Motzkin numbers ([16], A001006). We refer to Donaghey, Shapiro [5] and Stanley [17] for several combinatorial classes enumerated by the Motzkin and Catalan numbers.

- Bijective prooffor $\mathcal{S}_{n}^{\text {irr }}(132)$.

Here we construct a bijection between $\mathcal{S}_{n}^{\text {irr }}(132)$ and the set of Motzkin paths of length $n-1$. We define the map $f$ that transforms a permutation $\sigma=\sigma_{1} \sigma_{2} \cdots \sigma_{n} \in \mathcal{S}_{n}^{i r r}(132)$ into the Motzkin path $M$ of length $n-1$ obtained by the following process:

For each $i$ from 1 to $n-1$, 
(a) if $\sigma_{i}<\sigma_{i+1}$, then the $i$-th step of $M$ is a down step $D$;

(b) if $\sigma_{i}>\sigma_{i+1}$ and there exists $j>i+1$ such that $\sigma_{j}=\sigma_{i}+1$, then the $i$-th step of $M$ is an up step $U$;

(c) otherwise, the $i$-th step of $M$ is an horizontal step $H$.

For instance, the permutations $21,321,213,4213,3214,3241$ and 4321 are respectively transformed by $f$ into the Motzkin paths $H, H H, U D, H U D, U H D, U D H, H H H$ (see Figure 1 for an example with $n=14$ ).

Let us prove that $f$ is a one-to-one correspondence between $\mathcal{S}_{n}^{\text {irr }}(132)$ and the set of Motzkin paths of length $(n-1)$. Let $\sigma$ be a permutation of $\mathcal{S}_{n}^{i r r}(132)$. Let us take $i, 1 \leq i \leq n-1$, such that $(b)$ is verified. Then, there exists $j>i+1$ such that $\sigma_{j}=\sigma_{i}+1$. Since $\sigma$ avoids $132, \sigma_{j-1}<\sigma_{j}$ and the index $j-1>i$ verifies the case $(a)$. Conversely, if $i, 1 \leq i \leq n-1$, satisfies $(a)$ then $\sigma_{i}<\sigma_{i+1}$. Since $\sigma$ is irreducible, we have $\sigma_{i+1} \neq \sigma_{i}+1$. Thus, there exists $j \neq i$ and $j \neq i+1$ such that $\sigma_{j}=\sigma_{i+1}-1$. As $\sigma$ avoids 132 , we necessarily have $j<i$ and $j$ verifies $(b)$. Hence, there is a one-to-one correspondence between up steps and down steps such that each up step is associated with a down step on its right. This is precisely the characterization of the Motzkin paths.

Conversely, let $M$ be a Motzkin path of length $(n-1)$ and let us prove that there exists a permutation $\sigma \in \mathcal{S}_{n}^{i r r}(132)$ such that $f(\sigma)=M$. We proceed by induction on $n$ in order to construct the permutation $\sigma \in \mathcal{S}_{n}^{\text {irr }}(132)$.

We distinguish two cases: (1) $M=m H m^{\prime}$ where $m$ and $m^{\prime}$ are two Motzkin paths and such that $m$ does not contain any horizontal steps on the $x$-axis, and (2) $M=m U m^{\prime} D$ where $m$ and $m^{\prime}$ are two Motzkin paths such that $m$ does not belong to the case (1).

- (1) If $M=m H^{\prime}$, then we have $M=f(\sigma)$ where $\sigma=\beta n \gamma$ such that $\gamma(\operatorname{resp} . \operatorname{red}(\beta n))$ is recursively obtained from the Motzkin path $m^{\prime}$ (resp. $m$ ). Notice that the position of $n$ in $\sigma$ creates the horizontal step $H$ between $m$ and $m^{\prime}$.

- (2) If $M=m U m^{\prime} D$, then we have $M=f(\sigma)$ where $\sigma=\beta(n-1) \gamma n$ such that $\gamma(\operatorname{resp}$. $\operatorname{red}(\beta(n-$ $1)$ )) is recursively obtained from the Motzkin path $m^{\prime}$ (resp. $m$ ). Since $m$ does not belong to (1), its associated permutation necessarily have its greatest element on the last position. We conclude with a simple induction.

Notice that paths of type (1) are mapped to permutations whose last element is not $n$, and paths of type (2) are mapped to permutations that end with $n$.

For example, if $M=m U m^{\prime} D$ with $m=U H D$ and $m^{\prime}=U D H(n=9)$, then $\beta 8=7658$, $\operatorname{red}(\beta 8)=3214, \gamma=3241$ and $\sigma=765832419$. If $M=m H m^{\prime}$ with $m=U H D$ and $m^{\prime}=U D H$ $(n=8)$, then $\beta 8=7658, \operatorname{red}(\beta 8)=3214, \gamma=3241$ and $\sigma=76583241$.

Finally, the map $f$ is a one-to-one correspondence between $\mathcal{S}_{n}^{\text {irr }}(132)$ and the set of Motzkin paths of length $(n-1)$.

- Bijective proof for $\mathcal{S}_{n}^{\text {irr }}(321)$.

Here we construct a bijection between $\mathcal{S}_{n}^{\text {irr }}(321)$ and the set of Motzkin paths of length $n-1$. Let $\sigma=\sigma_{1} \sigma_{2} \cdots \sigma_{n}$ be a permutation in $\mathcal{S}_{n}^{\text {irr }}(321)$, and for $i \in[n]$ we denote by $s_{i}$ the maximum of the set $\left\{\sigma_{1}, \sigma_{2}, \ldots, \sigma_{i}\right\}$. We define the map $g$ that transforms a permutation $\sigma \in \mathcal{S}_{n}^{\text {irr }}(321)$ into the Motzkin path $M$ of length $n-1$ obtained by the following process:

For each $i$ from 1 to $n-1$, 


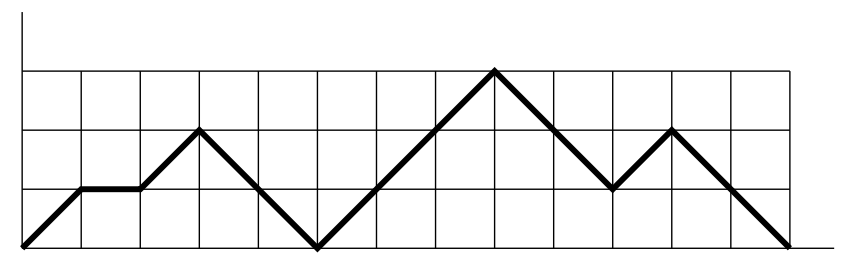

Fig. 1: The Motzkin path $U H U D D U U U D D U D D$ and its corresponding permutations $\sigma=$ (12) (11) 98 (10) (13) 5324617 (14) and $\sigma^{\prime}=2416358$ (10) (12) 79 (14) (11) (13) lying respectively in $\mathcal{S}_{14}^{\text {irr }}(132)$ and $\mathcal{S}_{14}^{\text {irr }}(321)$.

(a) if $s_{i}+1<\sigma_{i+1}$, then the $i$-th step of $M$ is an up step $U$;

(b) if there exists $j<i$ such that $\sigma_{i+1}=s_{j}+1$ and $s_{j}+1<\sigma_{j+1}$, then the $i$-th step of $M$ is a down step $D$;

(c) otherwise, the $i$-th step of $M$ is an horizontal step $H$.

For instance, the permutations $21,132,213,1324,3142,2143$ and 2413 are respectively transformed by $g$ into the Motzkin paths $H, U D, H H, U D H, H U D, H H H, U H D$ (see Figure 1 for an example of length 14).

Let us prove that $g$ is a one-to-one correspondence between $\mathcal{S}_{n}^{\text {irr }}(321)$ and the set of Motzkin paths of length $(n-1)$. Let $\sigma$ be a permutation of $\mathcal{S}_{n}^{i r r}(321)$. Let us take $i, 1 \leq i \leq n-1$, such that $(a)$ is verified. Since $s_{i}+1<\sigma_{i+1}$, there exists $j>i+1$, such that $\sigma_{j}=s_{i}+1$. A consequence is that the index $j-1, i<j-1<n$, verifies (b). Conversely, let us take $i, 1 \leq i \leq n-1$, satisfying (b). So there is $j<i$ such that $\sigma_{i+1}=s_{j}+1$ and $s_{j}+1<\sigma_{j+1}$ and the index $j$ verifies $(a)$. Thus, there is a one-to-one correspondence between up steps and down steps such that each up step is associated with a down step on its right. This is precisely the characterization of the Motzkin paths.

Conversely, let $M$ be a Motzkin path of length $(n-1)$ and let us prove that there exists a permutation $\sigma \in \mathcal{S}_{n}^{i r r}(321)$ such that $g(\sigma)=M$. We proceed by induction on $n$ in order to construct the permutation $\sigma \in \mathcal{S}_{n}^{i r r}(321)$.

We distinguish four cases: (1) $M=U^{k} D m$; (2) $M=U^{k} H^{\ell} m$; (3) $M=H^{k} U m$; and (4) $M=$ $H H \ldots H$, with $k \geq 1, \ell \geq 0$, and where $m$ is a suffix of the Motzkin path $M$.

- If $M=U^{k} D m$, then we have $M=g(\sigma)$ where $\sigma$ is recursively obtained from the permutation $\pi$ such that $g(\pi)=U^{k-1} H m$ by adding 1 on the left of $\pi$ and after increasing by one all values of $\pi$. Notice that $\pi$ cannot begin with 1 since there would be a down step just after $U^{k-1}$. Thus $\sigma$ does not contain any successions and avoids 321 .

- If $M=U^{k} H^{\ell} m$ where $m$ does not begin with $H$.

If $\ell=1$, then we have $M=g(\sigma)$ where $\sigma$ is recursively obtained from the permutation $\pi$ such that $g(\pi)=U^{k} m$ by inserting 1 between the $(k+1)$ and $(k+2)$-th positions of $\pi$ and after increasing by one all values of $\pi$ greater or equal than 1 . Since $g(\pi)=U^{k} m$ and $m$ does not start with $H$, we have $\pi_{k+2} \neq 1$ and the previous insertion of 1 does not create any succession and any pattern 321 .

If $M=U^{k} H^{\ell} m$ where $m$ does not begin with $H$ and with $\ell \neq 1$, then we have $M=g(\sigma)$ where $\sigma$ is recursively obtained from the permutation $\pi$ such that $g(\pi)=U^{k} H^{\ell-1} m$ by inserting $\pi_{k+\ell-1}+1$ 
between the $(k+\ell)$ and $(k+\ell+1)$-th positions of $\pi$ and after increasing by one all values of $\pi$ greater or equal than $\pi_{k+\ell-1}+1$.

Since the first step of $m$ is not $H$, we necessarily have either $\pi_{k+\ell+1}>s_{k+\ell}+1$ or $\pi_{k+\ell+1}<s_{k+\ell}$ which implies that the insertion of $\pi_{k+\ell-1}+1$ does not create a succession at position $(k+\ell+1)$ in $\sigma$. Also, the insertion of $\pi_{k+\ell-1}+1$ between the $(k+\ell)$ and $(k+\ell+1)$-th positions cannot create a succession at position $(k+\ell)$. So, the permutation $\sigma$ does not contain any succession.

Let us assume $s_{k+\ell}=\pi_{k+\ell}$. Since the $(k+\ell-1)$-th step is $H$, there is $j<k+l-1$ such that $\pi_{j}+1=\pi_{k+\ell}$. Therefore, the insertion of $\pi_{k+\ell-1}+1$ between the $(k+\ell)$ and $(k+\ell+1)$-th positions does not create a pattern 321 (since $\pi$ does not contain any pattern 321).

Now, let us assume that $s_{k+\ell} \geq \pi_{k+\ell}+1$. If we have $\pi_{k+\ell-1}=s_{k+\ell}$ then the insertion of $s_{k+\ell}+1$ between the $(k+\ell)$ and $(k+\ell+1)$-th positions cannot create a pattern 321. If we have $\pi_{k+\ell-1} \neq s_{k+\ell}$, we necessarily have $\pi_{k+\ell-1}<\pi_{k+\ell}$ and all values lying in [1, $\left.\pi_{k+\ell-1}\right]$ appear at positions $j<k+\ell$. So, the insertion of $s_{k+\ell}+1$ between the $(k+\ell)$ and $(k+\ell+1)$-th positions cannot create a pattern 321 . Finally, the permutation $\sigma$ belongs to $\mathcal{S}_{n}^{\text {irr }}(321)$. $U m$.

- If $M=H^{k} U m$, then we have $M=g(\sigma)$ where $\sigma=\pi u \pi^{\prime}$ such that $g(\pi u)=H^{k}$ and $g\left(\operatorname{red}\left(u \pi^{\prime}\right)\right)=$

- If $M=H^{k}$, then we have $M=g(\sigma)$ where $\sigma$ is recursively obtained from the permutation $\pi$ such that $g(\pi)=H^{k-1}$ by inserting $\pi_{k-1}+1$ in the last position and after increasing by one all values of $\pi$ greater or equal than $\pi_{k-1}+1$.

Below, we give an example for each previous case.

- If $M=U^{k} D m$ with $k=2, m=U H D D$, then $\pi=3517246, g(\pi)=U H U H D D$ and $\sigma=$ 14628357;

- if $M=U^{k} H^{\ell} m$ with $k=2, \ell=3$ and $m=D D$, then $\pi=2461735, g(\pi)=U U H H D D$ and $\sigma=35718246$;

- if $M=H^{k} U m$ with $k=3, m=D$, then $u=2, \pi u=3142, g(\pi u)=H H H, u \pi^{\prime}=265$, $g(132)=U D$ and $\sigma=314265$;

- if $M=H^{k}$ with $k=5$, then $\pi=31425, g(\pi)=H H H H$ and $\sigma=415263$.

The bijection $f$ (resp. $g$ ) from $\mathcal{S}_{n}^{\text {irr }}(132)$ (resp. $\left.\mathcal{S}_{n}^{\text {irr }}(321)\right)$ to the set of Motzkin paths of length $(n-1)$ induces a new constructive bijection between Dyck paths and some restricted irreducible permutations:

Corollary 1 Let $P_{2 n+1}$ be the set of permutations $\sigma \in \mathcal{S}_{2 n+1}^{i r r}(132)$ such that for all $i, 1 \leq i \leq 2 n-1$, with $\sigma_{i}>\sigma_{i+1}$ there exists $j>i+1$ with $\sigma_{j}=\sigma_{i}+1$. Then the map $f$ induces a constructive bijection between $P_{2 n+1}$ and the set of Dyck Paths of length $2 n$.

Corollary 2 Let $P_{2 n+1}^{\prime}$ be the set of permutations $\sigma \in \mathcal{S}_{2 n+1}^{\text {irr }}(321)$ such that $\sigma_{1}=1$ and $\sigma_{i+1}-$ $\max \left\{\sigma_{1}, \ldots, \sigma_{i}\right\} \leq 2$ and $\sigma_{i+1}-\max \left\{\sigma_{1}, \ldots, \sigma_{i}\right\} \neq 1$ for all $1 \leq i \leq 2 n-1$. The map $g$ induces a constructive bijection between $P_{2 n+1}^{\prime}$ and the set of Dyck Paths of length $2 n$.

For example, we have $P_{3}=\{213\}, P_{5}=\{42135,32415\}, P_{7}=\{6421357,5462137,6324157$, $5324617,4352617\}, P_{3}^{\prime}=\{132\}, P_{5}^{\prime}=\{13254,13524\}$ and $P_{7}^{\prime}=\{1325476,1325746,1352476,1352746$, $1357246\}$. 


\section{Avoiding two patterns of length 3}

In this section, we explore the avoidance of two patterns of length 3 for irreducible permutations. All enumerative results of this section are listed in Table 2

Theorem 4 For $\alpha \in\{231,312\}$, the sets $\mathcal{S}_{n}^{\text {irr }}(321, \alpha), n \geq 1$, are enumerated by the Padovan's spiral numbers defined by the generating function $\frac{1+x}{1-x^{2}-x^{3}}$ (see [16], A134816).

Proof: Let $\sigma=\beta n \gamma$ be a permutation in $\mathcal{S}_{n}^{\text {irr }}(321, \alpha)$ where $\beta$ and $\gamma$ are two subsequences of $[n-1]$. Since $\sigma$ avoids 321, $\gamma=\gamma_{1} \gamma_{2} \cdots \gamma_{k}$ is an increasing subsequence (possibly empty) of $[n-1]$. We distinguish two cases: (1) $\gamma$ is empty; and (2) $\gamma$ is not empty.

Case (1). This means that $\beta \in \mathcal{S}_{n-1}^{\text {irr }}(321, \alpha)$ and the last entry of $\beta$ is different from $n-1$. Conversely, if we add $n$ to the right of a permutation $\pi$ in $\mathcal{S}_{n-1}^{i r r}(321, \alpha)$ such that $\pi_{n-1} \neq n-1$, we obtain a permutation of $\mathcal{S}_{n}^{\text {irr }}(321, \alpha)$.

Case (2). Now $\gamma=\gamma_{1} \gamma_{2} \cdots \gamma_{k}$ is not empty. For a contradiction, let us assume $k \geq 2$. Then, $\sigma$ contains the pattern 312. Since $\sigma$ is irreducible, there is an element $b$ of $\beta$ such that $\gamma_{k-1}<b<\gamma_{k}<n$ which implies that $\sigma$ contains the pattern 231. Thus, we necessarily have $k=1$. In this case, $\sigma$ can be written $\beta n(n-1)$ where $\beta \in \mathcal{S}_{n-2}^{\text {irr }}(321, \alpha)$.

Let $f(x)$ (resp. $g(x)$ ) be the generating function for permutations $\sigma \in \mathcal{S}_{n}^{\text {irr }}(321, \alpha)$ (resp. $\sigma \in$ $\mathcal{S}_{n}^{i r r}(321, \alpha)$ such that $\left.\sigma_{n} \neq n\right)$. The previous study induces that $f(x)=1+x \cdot g(x)+x^{2} \cdot f(x)$ with $g(x)=f(x)-x \cdot g(x)$. We obtain $g(x)=\frac{1}{1-x^{2}-x^{3}}$ and $f(x)=\frac{1+x}{1-x^{2}-x^{3}}=\frac{1}{1-x} \cdot h(x)$ where $h(x)$ is the generating function for the Padovan numbers (see [16], A000931). This corresponds to the Padovan's spiral numbers [16], A134816).

Notice that this last proof shows that the three sets $\mathcal{S}_{n}^{\text {irr }}(321,231), \mathcal{S}_{n}^{\text {irr }}(321,312)$ and $\mathcal{S}_{n}^{\text {irr }}(321,231,312)$ are identical, not just equinumerous. To my knowledge, there is no analogous result in the enumeration of general (as opposed to irreducible) permutations.

Theorem 5 For $\alpha \in\{132,213\}$, the set $\mathcal{S}_{n}^{\text {irr }}(321, \alpha)$ is empty for $n \geq 4$. The set $\mathcal{S}_{n}^{\text {irr }}(321,123)$ is empty for $n \geq 5$.

Proof: The result is well known for $\alpha=123$. Now, let us take $\alpha=213$ (the case $\alpha=132$ will be obtained with a simple Wilf equivalence). Let $\sigma$ be a permutation of $\mathcal{S}_{n}^{\text {irr }}(321,213)$. Writing $\sigma=\beta n \gamma$ with $\beta$ and $\gamma$ are two subsequences of $[n-1]$, the avoidance of 213 implies that $\beta=\beta_{1} \cdots \beta_{k}$, where $\beta_{1}<\beta_{2}<\cdots<\beta_{k}$; the avoidance of 321 implies that $\gamma=\gamma_{1} \gamma_{2} \ldots \gamma_{\ell}$, where $\gamma_{1}<\gamma_{2}<\cdots<\gamma_{\ell}$. Since $\sigma$ is irreducible and avoids 213, $\gamma$ and $\beta$ contain at most one element which implies that there does not exist any permutations $\sigma$ in $\mathcal{S}_{n}^{\text {irr }}(321,213)$ whenever $n \geq 4$.

Theorem 6 For $n \geq 1$, the set $\mathcal{S}_{n}^{\text {irr }}(213,132)$ is reduced to the unique permutation $n(n-1) \cdots 321$.

Proof: Let $\sigma$ be a permutation in $\mathcal{S}_{n}^{\text {irr }}(213,132)$. We can write $\sigma=\beta n \gamma$ where $\beta$ and $\gamma$ are two subsequences of $[n-1]$. Since $\sigma$ avoids 213 , we have $\beta=\beta_{1} \beta_{2} \cdots \beta_{k}$, where $\beta_{1}<\beta_{2}<\cdots<\beta_{k}$. Since $\sigma$ is irreducible, we have $\beta_{k} \neq n-1$, and thus $n-1$ lies in $\gamma$. Since $\gamma$ avoids $132, \beta$ is empty (otherwise, $\beta_{1} n(n-1)$ would be a pattern 132). Thus we have $\sigma=n \gamma$. A straightforward induction provides $\sigma=n(n-1) \cdots 321$. 
Theorem 7 The sets $\mathcal{S}_{n}^{\text {irr }}(231,312), n \geq 0$, are enumerated by the Tribonacci numbers (see [16], A000213).

Proof: Let $\sigma$ be a permutation in $\mathcal{S}_{n}^{\text {irr }}(231,312)$. Since $\sigma$ avoids 312, it can be written $\sigma=\beta n \gamma$ where $\beta$ and $\gamma$ are two subsequences of $[n-1]$ such that either $\gamma=\gamma_{1} \cdots \gamma_{k}$ with $\gamma_{1}>\gamma_{2}>\cdots>\gamma_{k}$ or $\gamma$ is empty. Since $\gamma$ avoids 231, $\beta$ does not contain any values greater than $\gamma_{k}$. Thus we have either $\gamma$ is empty or $\gamma=\beta n(n-1)(n-2) \cdots(n-k)$ where $\beta \in \mathcal{S}_{n-k-1}^{\text {irr }}(231,312)$.

Let $f(x)$ (resp. $g(x)$ ) be the generating function for $\mathcal{S}_{n}^{\text {irr }}(231,312), n \geq 0$, (resp. for $\mathcal{S}_{n}^{\text {irr }}(231,312)$, $n \geq 1$, with the restriction that permutations do not end with $n)$. Then we have $f(x)=1+x+x g(x)+$ $g(x)$. The above structure of a permutation in $\mathcal{S}_{n}^{\text {irr }}(231,312)$ ensures that $g(x)=\left(\frac{1}{1-x}-1-x\right) \cdot f(x)$. Thus we have $f(x)=\frac{1-x^{2}}{1-x-x^{2}-x^{3}}$ that corresponds to the Tribonacci numbers [16], $A 000213$.

Theorem 8 For $\alpha \in\{231,312\}$ and $\beta \in\{132,213\}$, the sets $\mathcal{S}_{n}^{\text {irr }}(\alpha, \beta), n \geq 1$, are enumerated by the Fibonacci numbers (see [16], A000045).

Proof: Let $\sigma=\sigma_{1} \sigma_{2} \cdots \sigma_{n}$ be a permutation in $\mathcal{S}_{n}^{i r r}(231,213)$. Since $\sigma$ avoids 231 and 213, then $\sigma_{i}$, $1 \leq i \leq n$, is either the smallest or the greatest element of the set $\left\{\sigma_{i}, \sigma_{i+1}, \ldots, \sigma_{n}\right\}$. We deduce that $\sigma$ can be written either $\sigma=1 n \gamma$ or $\sigma=n \gamma$, where $\gamma$ avoids 231 and 213. If $a_{n}=\operatorname{card}\left(\mathcal{S}_{n}^{i r r}(231,213)\right)$ then $a_{1}=1, a_{2}=1$, and $a_{n}=a_{n-1}+a_{n-2}$ which defines the Fibonacci numbers.

The classical symmetries deal with the remaining cases.

Theorem 9 For $\alpha \in\{312,231\}$, the sets $\mathcal{S}_{n}^{\text {irr }}(123, \alpha), n \geq 1$, are enumerated by the Triangular numbers $\frac{n(n-1)}{2}$ (see [16], A000217).

Proof: Let $\sigma=\sigma_{1} \sigma_{2} \cdots \sigma_{n}$ be a permutation in $\mathcal{S}_{n}^{\text {irr }}(123,312)$. Since $\sigma$ avoids 123 and 312, $\sigma$ can be written $\sigma=k(k-1) \cdots \ell n \gamma$ where $1 \leq \ell \leq k \leq n$ and $\gamma$ is a decreasing sequence of integers. For $k$ fixed, $1 \leq k \leq n-2$, there are $k$ possible permutations obtained whenever $\ell$ describes $[1, k]$. For $k=n-1$, the permutation $(n-1) n(n-2) \cdots 21$ is not irreducible, thus we do not consider it. In this case, there are $(n-2)$ possible permutations. Finally, $\mathcal{S}_{n}^{i r r}(123,312)$ is enumerated by $1+2+3+\cdots+$ $(n-2)+(n-2)+1=\frac{n(n-1)}{2}$ which is the Sloane's sequence A000217. A simple symmetry gives the result for $\alpha=231$.

Theorem 10 For $\alpha \in\{132,213\}$, the sets $\mathcal{S}_{n}^{\text {irr }}(123, \alpha), n \geq 1$, are enumerated by the sequence [16], A005251.

Proof: Let $\sigma=\sigma_{1} \sigma_{2} \cdots \sigma_{n}$ be a permutation in $\mathcal{S}_{n}^{\text {irr }}(123,132)$. Since $\sigma$ avoids 123 and $132, \sigma_{1}$ is either $(n-1)$ or $n$. In the case where $\sigma_{1}=n$, then $\sigma$ can be written $\sigma=n \gamma$ where $\gamma \in \mathcal{S}_{n-1}^{\text {irr }}(123,132)$. In the case where $\sigma_{1}=n-1, \sigma$ can be written: $\sigma=(n-1)(n-2) \cdots(n-k) n \gamma, 2 \leq k \leq n-1$, where $\gamma$ belongs to $\mathcal{S}_{n-1-k}^{\text {irr }}(123,132)$. If $a_{n}=\operatorname{card}\left(\mathcal{S}_{n}^{\text {irr }}(123,132)\right)$ then we deduce $a_{n}=a_{n-1}+a_{n-3}+$ $a_{n-4}+\cdots+a_{1}+1$ where $a_{1}=1$ and $a_{2}=1$ which corresponds to the Sloane's sequence A005251. The case $\alpha=213$ is obtained by symmetry. 


\begin{tabular}{|c|c|c|}
\hline Pattern & Sloane & Sequence \\
\hline$\{321,231\},\{321,312\}$ & $A 134816$ & Padovan's spiral \\
\hline$\{321,132\},\{321,213\}$ & & $1,1,1,0,0,0, \ldots$ \\
\hline$\{321,123\}$ & & $1,1,2,3,0,0, \ldots$ \\
\hline$\{213,132\}$ & & $1,1,1,1, \ldots$ \\
\hline$\{231,312\}$ & $A 000213$ & Tribonacci \\
\hline$\{231,132\},\{231,213\},\{312,132\},\{312,213\}$ & $A 000045$ & Fibonacci \\
\hline$\{123,231\},\{123,312\}$ & $A 000217$ & $\frac{n(n-1)}{2}$ \\
\hline$\{123,132\},\{123,213\}$ & $A 005251$ & $1,1,2,4,7,12,21,37,65$ \\
\hline
\end{tabular}

Tab. 2: Wilf-equivalence classes for two pattern subsets of $S_{3}$ in irreducible permutations.

\section{Fixed point free involutions}

In this section, we study the avoidance of one pattern of length 3 for fixed point free irreducible involutions, i.e., for involutions with no fixed points and no successions. All enumerative results of this section are listed in Table 3. Let $\mathcal{D} \mathcal{I}$ (resp. $\mathcal{D} \mathcal{I}^{i r r}$ ) be the set of fixed point free (resp. fixed point free irreducible) involutions. These sets restricted to their length $n$ elements will be respectively denoted $\mathcal{D} \mathcal{I}_{n}$ and $\mathcal{D} \mathcal{I}_{n}^{\text {irr }}$.

\subsection{Enumerative results}

Theorem 11 The sets $\mathcal{D I}_{n}^{i r r}, n \geq 0$, are enumerated by the sequence $a_{n}$ defined by $a_{0}=0, a_{2}=1$, $a_{2 n}=(2 n-2) a_{2 n-2}+(2 n-4) a_{2 n-4}$ for $n \geq 2$ and $a_{2 n+1}=0$ for $n \geq 0$ (see [16], A165968).

Proof: Obviously, there does not exist any fixed point free involutions of odd length; thus $a_{2 n+1}=0$ for $n \geq 0$. Now, let $\sigma$ be an irreducible involution of length $2 n, n \geq 2$, with no fixed points. Let $k$ be the index in $[2 n-1]$ such that $\sigma_{k}=2 n$ (and also $\sigma_{2 n}=k$ ).

Let $\pi$ be the involution of length $(2 n-2)$ obtained from $\sigma$ by deleting $k$ and $2 n$, and reducing the result to an involution of length $(2 n-2)$. Two cases occur: $(a) \pi$ is irreducible; and $(b) \pi$ has the two successions $\left(\pi_{k-1}, \pi_{k}\right)$ and $(k-1, k)$. Conversely, each irreducible involution of length $2 n$ can be obtained from an involution $\pi$ belonging to one of the two previous cases $(a)$ and $(b)$.

Thus, the generating function for the permutations $\sigma$ of type $(a)$ is $(2 n-2) a_{2 n-2}$.

Now let us consider involutions of type $(b)$. Let $\mathcal{A}_{2 n-2}$ be the set of involutions of length $(2 n-2)$ with exactly two successions and with no fixed points. An involution in $\mathcal{A}_{2 n-2}$ can be extended into an irreducible involution of length $2 n$ of type $(b)$ in two possible ways. So, let $\pi$ be an involution in $A_{2 n-2}$ such that $\left(\pi_{k}, \pi_{k+1}\right)$ and $(k, k+1)$ are the two successions. From $\pi$, we construct the pair $\left(k, \pi^{\prime}\right) \in[2 n-4] \times \mathcal{D} \mathcal{I}_{2 n-4}^{\text {irr }}$ where $\pi^{\prime}$ is obtained from $\pi$ by deleting the two entries $\pi_{k+1}$ and $(k+1)$, and reducing the result to a permutation of length $(2 n-4)$. Since we obtain the same permutation in $\pi^{\prime} \in \mathcal{D} \mathcal{I}_{2 n-4}^{i r r}$ by deleting $\pi_{k}$ and $k$, the cardinality of $\mathcal{A}_{2 n-2}$ is half the cardinality of $[2 n-4] \times \mathcal{D} \mathcal{I}_{2 n-4}^{i r r}$. Therefore, the generating function for the number of involutions of type $(b)$ is given by $2 \cdot \frac{(2 n-4) \cdot a_{2 n-4}}{2}$. 
Finally, we conclude $a_{2 n}=(2 n-2) a_{2 n-2}+(2 n-4) a_{2 n-4}$ for $n \geq 2$.

More generally, the proofs of Lemmas 1 and 2 imply that there are $\frac{n-1}{k} \cdot a_{2 n}$ fixed point free involutions of length $2 n$ with exactly $2 k$ successions, where $a_{2 n}$ is the sequence defined in Theorem 11 .

Theorem 12 For $\alpha \in\{132,213,321\}$ and $n \geq 0$, the sets $\mathcal{D I}_{2 n}^{\text {irr }}(\alpha)$ of irreducible involutions of length $2 n$ without fixed points and avoiding the pattern $\alpha$, are enumerated by the Motzkin numbers (see [16], A001006). The generating function for $\mathcal{D} \mathcal{I}_{n}^{i r r}(\alpha)$ is given by $\frac{1+x^{2}-\sqrt{\left(1-3 x^{2}\right)\left(1+x^{2}\right)}}{2 x^{2}}$.

Proof: Since the set $\mathcal{D I}(\alpha)$ is expanded and closed under deflation, we apply Lemma 2 of Section 2. As the generating function for $\mathcal{D} \mathcal{I}_{n}(\alpha), n \geq 0$, is $f(x)=c\left(x^{2}\right)$, where $c(x)=\frac{1-\sqrt{1-4 x}}{2 x}$ is the generating function for the Catalan numbers, the generating function for $\mathcal{D I}_{n}^{i r r}(\alpha)$ is

$$
g(x)=f\left(\frac{x}{\sqrt{1+x^{2}}}\right)=c\left(\frac{x^{2}}{1+x^{2}}\right)=\frac{1+x^{2}-\sqrt{\left(1-3 x^{2}\right)\left(1+x^{2}\right)}}{2 x^{2}} .
$$

A simple application of Lemma 2 yields the bivariate generating function $f(x, y)=c\left(\frac{x^{2}}{1+(1-y) x^{2}}\right)$ of the set $\mathcal{D} \mathcal{I}(\alpha)$ of fixed point free involutions avoiding the pattern $\alpha$ for $\alpha \in\{321,213,132\}$, where the coefficient of $x^{n} y^{k}$ gives the number of elements in $\mathcal{D} \mathcal{I}_{n}(\alpha)$ with exactly $k$ successions.

Theorem 13 For $\alpha \in\{231,312\}$ and $n \geq 0$, the sets $\mathcal{D I}_{2 n}^{\text {irr }}(\alpha)$ of irreducible involutions of length $2 n$ without fixed points and avoiding the pattern $\alpha$, are enumerated by $2^{n-1}$.

Proof: Let $\alpha=231$ and $\sigma=\sigma_{1} \sigma_{2} \cdots \sigma_{2 n}$ be an irreducible involution of length $2 n$ without fixed points and avoiding $\alpha$. Since $\sigma$ avoids 231, we can decomposed $\sigma=\beta(2 n) \gamma(2 k+1)$ where $2 k+1 \leq 2 n-1, \beta \in$ $\mathcal{D I}_{2 k}^{i r r}(\alpha)$ and $(2 n) \gamma(2 k+1)$ is a decreasing sequence of consecutive numbers in the interval $[2 k+1,2 n]$. Therefore, if $a_{2 n}$ enumerates the set $\mathcal{D} \mathcal{I}_{2 n}^{\text {irr }}(\alpha)$, we deduce the induction $a_{2 n}=a_{0}+a_{2}+a_{4}+\cdots+a_{2 n-2}$ where $a_{0}=1$. We obtain $a_{2 n}=2^{n-1}$ for $n \geq 1$ with $a_{0}=1$. The case $\alpha=312$ is handled by the symmetry $\sigma \rightarrow \sigma^{-1}$.

Theorem 14 The generating function for the sets $\mathcal{D} \mathcal{I}_{n}^{\text {irr }}(123), n \geq 0$, of irreducible involutions of length $n$ without fixed points and avoiding the pattern 123 is

$$
\frac{2 x^{2}\left(x^{2} \sqrt{1-4 x^{2}}-1\right)}{\sqrt{1-4 x^{2}} \cdot\left(1+2 x^{4}+\sqrt{1-4 x^{2}}\right)} .
$$

Proof: Let $\sigma$ be an involution of length $2 n$ without fixed points containing at least one succession. We suppose that $\left(\sigma_{k}, \sigma_{k+1}\right)$ is the leftmost succession. Since $\sigma$ avoids 123 , it is straightforward to see that $\sigma_{k}=2 n-k$ and $\sigma_{k+1}=2 n-k+1$.

Then $\sigma$ can be written: $\sigma=\lambda(2 n-k)(2 n-k+1) \beta k(k+1) \gamma$ where $\lambda$ (resp. $\gamma$ ) is a 123 -avoiding sequence of elements in $[2 n-k+2,2 n]$ (resp. $[1, k-1]$ ) without successions, such that $\operatorname{red}(\lambda)=\gamma^{-1}$ and $\beta$ is a sequence of elements in $[k+2,2 n-k-1]$ where $\operatorname{red}(\beta)$ is an involution of length $2 n-2 k-2$ without fixed points. See Figure 2 for an illustration of the structure of $\sigma$. 
Let $g(x)=\frac{\frac{1}{1-4 x^{2}}-1}{2}$ be the generating function for the set of involutions without fixed points and avoiding 123 (see for instance [4]). Let $f(x)$ be the generating function of irreducible involutions without fixed points and avoiding 123 . We have

$$
g(x)-f(x)=x^{4} \cdot h\left(x^{2}\right) \cdot(g(x)+1)
$$

where $h(x)$ is the generating function for irreducible permutations avoiding the pattern 123 (see Theorem 11. Finally, a simple calculation gives the desired results.

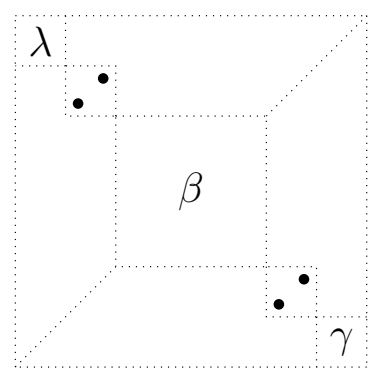

Fig. 2: Proof of Theorem 14 The special structure of a fixed point free involution avoiding 123 and with at least one succession.

\begin{tabular}{|c|c|c|c|}
\hline Pattern & Sequence & Sloane & $a_{2 n}, n \geq 1$ \\
\hline\{\} & $\left\{\begin{array}{l}a_{2 n+1}=0 \\
a_{2 n}=(2 n-2) a_{2 n-2}+(2 n-4) a_{2 n-4}\end{array}\right.$ & $A 165968$ & $1,2,10,68,604$ \\
\hline$\{132\},\{213\},\{321\}$ & Motzkin & $A 001006$ & $1,1,2,4,9,21$ \\
\hline$\{231\},\{312\}$ & $2^{n-1}$ & $A 000079$ & $1,2,4,8,16,32$ \\
\hline$\{123\}$ & $\frac{2 x^{2}\left(x^{2} \sqrt{1-4 x^{2}}-1\right)}{\sqrt{1-4 x^{2}} \cdot\left(1+2 x^{4}+\sqrt{1-4 x^{2}}\right)}$ & New & $1,2,8,30,109,401$ \\
\hline
\end{tabular}

Tab. 3: Fixed point free irreducible involutions avoiding at most one pattern of $\mathcal{S}_{3}$.

\subsection{Bijective proofs}

Recall that a Motzkin path of length $n$ is a lattice path starting at $(0,0)$, ending at $(n, 0)$, and never going below the $x$-axis, consisting of up steps $U=(1,1)$, horizontal steps $H=(1,0)$, and down steps $D=(1,-1)$.

- Bijective proof for $\mathcal{D} \mathcal{I}_{2 n}^{\text {irr }}(132)$.

A fixed point free irreducible involution $\sigma$ of length $2 n$ that avoids the pattern 132 is necessarily of the form $\sigma=\beta \beta^{\prime}$ where $\beta^{\prime}$ belongs to $\mathcal{S}_{n}^{i r r}(132)$ and such that $\operatorname{red}(\beta)=\beta^{\prime-1}$ (all values of $\beta$ are greater 
than those of $\left.\beta^{\prime}\right)$. Using the bijection $f$ from $\mathcal{S}_{n}^{\text {irr }}(132)$ to the set of Motzkin paths of length $(n-1)$ (see Section 3.2), we deduce immediately a constructive bijection $\bar{f}$ between $\mathcal{D I}_{2 n}^{i r r}(132)$ and the set of Motzkin paths of length $(n-1)$ as follows: $\bar{f}(\sigma)=f\left(\beta^{\prime}\right)$.

- Bijective proof for $\mathcal{D} \mathcal{I}_{2 n}^{\text {irr }}(321)$.

This part presents a constructive bijection between $\mathcal{D I}_{2 n}^{i r r}(321)$ and the set of Motzkin paths of length $(n-1)$.

Let $\sigma=\sigma_{1} \cdots \sigma_{2 n}$ be an involution of length $2 n$ without fixed points and that avoids 321. Then $\sigma$ is the product of $n$ transpositions (cycles of length 2): $\sigma=\left\langle\ell_{1}, r_{1}\right\rangle \cdot\left\langle\ell_{2}, r_{2}\right\rangle \cdots\left\langle\ell_{n}, r_{n}\right\rangle$ where $\ell_{1}<\ell_{2}<$ $\cdots<\ell_{n}, r_{1}<r_{2}<\cdots<r_{n}$ and $r_{i}<\ell_{i}$ for $1 \leq i \leq n$. In fact, the values $\ell_{i}$ (resp. $r_{i}$ ) for $1 \leq i \leq n$, are the left-to-right maxima (resp. right-to-left minima) of $\sigma$, i.e., $\ell_{i}$ (resp. $r_{i}$ ) is greater (resp. less) than all values on its left (resp. right) in $\sigma$.

Remark 2 It is well-known (see [4] for instance) that involutions of length $2 n$ without fixed points and avoiding 321 are enumerated by the $n$-th Catalan number. Such an involution is associated to the Dyck path from $(0,0)$ to $(2 n, 0)$ by the following process: reading $\sigma$ from left to right, we replace each leftto-right maxima with $U=(1,1)$ and each right-to-left minima with $D=(1,-1)$. For example, the involution $35172846109=\langle 3,1\rangle \cdot\langle 5,2\rangle \cdot\langle 7,4\rangle \cdot\langle 8,6\rangle \cdot\langle 10,9\rangle$ is associated to the Dyck path UUDUDUDDUD.

On the other hand, $\sigma$ can be viewed as a matching by putting $2 n$ points labeled from 1 to $2 n$ in this order, and then connecting, for $1 \leq j \leq n$, the numbers $\ell_{j}$ and $r_{j}$ by an arc (see Figure 4 for an illustration).

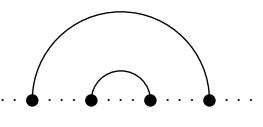

(1)

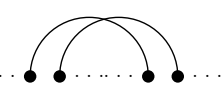

(2)

Fig. 3: The two forbidden configurations in the matching of an involution $\sigma \in \mathcal{D} \mathcal{I}_{2 n}^{i r r}(321)$.

Therefore, a fixed point free involution $\sigma$ avoids 321 means that its matching does not contain nesting arcs, i.e. the configuration (1) in Figure 3 does not occur. Moreover, $\sigma$ does not contain any successions if and only if the configuration (2) in Figure 3 does not occur, i.e., there does not exist two arcs $a_{1}$ and $a_{2}$ such that $a_{2}$ is obtained from $a_{1}$ by an horizontal translation of $(1,0)$. These two last conditions can also be expressed using the one-line notation of $\sigma$ as follows.

A fixed point free irreducible involution $\sigma$ avoids 321 if and only if, for $1 \leq i \leq n-1$, at least one of the two following statements is verified:

$\left(A_{i}\right)$ there exists a right-to-left minimum in $\sigma$ between the $i$-th and $(i+1)$-th left-to-right maxima;

$\left(B_{i}\right)$ there exists a left-to-right maximum between the $i$-th and $(i+1)$-th right-to-left minima.

Let us define the map $h$ that transforms $\sigma \in \mathcal{D I}_{2 n}^{i r r}(321)$ into the Motzkin path $M$ from $(0,0)$ to $(n-1,0)$ defined as follows:

For $i$ from 1 to $n-1$,

(a) if $B_{i}$ is verified but not $A_{i}$, then the $i$-th step of $M$ is an up step $U$; 

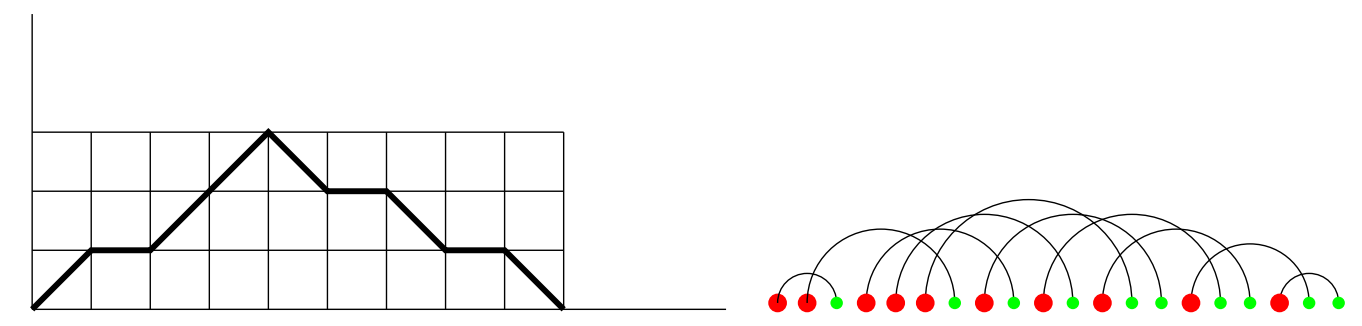

Fig. 4: The Motzkin path $U H U U D H D H D$ and its corresponding involution $\sigma=$ $3719(11)(13) 2(14) 4(16) 5(17) 68(19)(10)(12)(20)(15)(18)$.

(b) if $A_{i}$ is verified but not $B_{i}$, then the $i$-th step of $M$ is a down step $D$;

(c) if $A_{i}$ and $B_{i}$ are verified, then the $i$-th step of $M$ is an horizontal step $H$.

For instance, involutions 2143, 351624, 214365, 35172846, 35162487, 21573846 and 21436587 are respectively transformed by $h$ into $H, U D, H H, U H D, U D H, H U D$ and $H H H$ (see Figure 4 for an example with $2 n=20$ ).

Let us prove that $h$ is a one-to-one correspondence between $\mathcal{D I}_{2 n}^{i r r}(321)$ and the set of Motzkin paths of length $n-1$. Let $\sigma$ be an involution in $\mathcal{D I}_{2 n}^{i r r}(321)$. Using Remark 2 . $\sigma$ is associated with a Dyck path where left-to-right maxima (resp. right-to-left minima) correspond to up steps (resp. down steps). So, there is a one-to-one correspondence between up steps and down steps such that the image of an up step is a down step lying on its right. This induces a one-to-one correspondence $c$ between the set of all occurrences of $U U$ and the set of all occurrences of $D D$ such that the image by $c$ of a UU-occurrence lies on its right. This implies that there is a one-to-one correspondence $\bar{c}$ between the sets $U p=\{i, 1 \leq i \leq$ $n-1,(a)$ is verified $\}$ and Down $=\{i, 1 \leq i \leq n-1,(b)$ is verified $\}$ such that $\bar{c}(i)$ is greater than $i$ (we have $\bar{c}(i) \neq i$ since the configuration (2) of Figure 3 does not occur). As the function $h$ associates an up step when $i \in U p$, a down step when $i \in$ Down and an horizontal step otherwise, $h(\sigma)$ is a Motzkin path of length $(n-1)$. Moreover this construction ensures that the images by $h$ of two different involutions necessarily yields two different Motzkin paths.

Conversely, from any Motzkin path $M$ of length $n-1$, we construct an involution of length $2 n$ without fixed points and that avoids 321, by the following process. More precisely, we will construct a sequence of red and green points where red (resp. green) points correspond to left-to-right maxima (resp. right-to-left minima) of the involution of length $2 n$. This sequence will characterize the desired involution.

We start the process with a red point followed by a green point. Through the Motzkin path $M$ from left to right:

- if we meet an up step $U$, then we add one red point just after the last red point, and we add one red and one green points (in this order) on the right;

- if we meet a down step $D$, then we add one green point on the right;

- if we meet horizontal step $H$, then we add one red and one green points (in this order) on the right.

For instance, this process applying to the Motzkin path $M=U H D$ provides the following steps: 


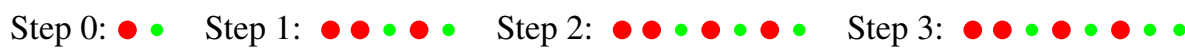

Fig. 5: Construction of the involution $\sigma=35172846$ from the Motzkin path $M=U H D$.

At each step of this process, we add only one green point on the right. Moreover, the number of red points is, at each step, at most the number of green points; at the end of the process, there is equality. The configuration (2) of Figure 3 does not occur. Therefore, the obtained matching corresponds to that of a fixed point free irreducible involution that avoids 321 and such that its image by $h$ is exactly the Motzkin path $M$. We conclude that $h$ is a one-to-one correspondence between $\mathcal{D I}_{2 n}^{i r r}(321)$ and the set of Motzkin paths of length $n-1$.

Notice that the above construction appears as a generalization of the bijection of P. Manara and C. Perelli Cippo, [9], which transforms a restricted set of Motzkin paths into the set of simple involutions avoiding the pattern 321 .

\section{Pattern avoiding involutions}

In this section, we present enumerative results for sets of irreducible involutions avoiding one pattern of length three (see Table 4).

Theorem 15 For $\alpha \in\{231,312\}$, the sets $\mathcal{I}_{n}^{\text {irr }}(\alpha), n \geq 0$, of irreducible involutions of length $n$ avoiding $\alpha$ are enumerated by the Tribonacci numbers (see [16], A000213).

Proof: A length $n$ irreducible involution $\sigma$ avoiding 231 can be written $\beta n \gamma k, 1 \leq k \leq n$, where $\beta \in \mathcal{I}_{k-1}^{\text {irr }}(\alpha)$ such that the last value of $\beta$ is different from $n-1$ whenever $k=n$, and where $n \gamma k=$ $n(n-1) \cdots(k+1) k$. Let $g(x)$ (resp. $h(x)$ ) be the generating function for the sets $\mathcal{I}_{n}^{\text {irr }}(\alpha), n \geq 0$, (resp. for the sets of irreducible involutions of length $n$ avoiding $\alpha$ such that the last value is different from $n$ ).

According to the above structure of $\sigma$, it is straightforward to see that $g(x)=1+x^{2} \frac{g(x)}{1-x}+x h(x)$ with $(1+x) h(x)=g(x)$. Thus we obtain $g(x)=\frac{x^{2}-1}{x^{3}+x^{2}+x-1}$ which is the generating function for the Tribonacci numbers. The case $\alpha=312$ is handled by the symmetry $\sigma \rightarrow \sigma^{-1}$.

Theorem 16 The generating function for the sets $\mathcal{I}_{n}^{\text {irr }}(123), n \geq 0$, of irreducible involutions of length $n$ avoiding 123 is given by $\frac{1-2 x-2 x^{2}+4 x^{3}+\sqrt{1-4 x^{2}}}{(1-2 x)\left(1+2 x^{4}+\sqrt{1-4 x^{2}}\right)}$.

Proof: Let $\sigma$ be an involution of length $n$ containing at least one succession. We suppose that $\left(\sigma_{k}, \sigma_{k+1}\right)$ is the leftmost succession. Since $\sigma$ avoids 123, it is straightforward to see that $\sigma_{k}=n-k$ and $\sigma_{k+1}=$ $n-k+1$. We distinguish two cases: either (1) $\sigma_{k} \neq k$ or (2) $\sigma_{k}=k$.

In the first case, $\sigma$ can be written $\sigma=\lambda(n-k)(n-k+1) \beta k(k+1) \gamma$ where $\lambda$ (resp. $\gamma$ ) is a 123-avoiding sequence of elements in $[n-k+2, n]$ (resp. $[1, k-1]$ ) without successions, such that $\operatorname{red}(\lambda)=\gamma^{-1}$ and $\beta$ is a sequence of elements in $[k+2, n-k-1]$ where $\operatorname{red}(\beta)$ is an involution of length $n-2 k-2$.

In the second case, $\sigma$ can be written $\sigma=\lambda k(k+1) \gamma$ where $\lambda$ (resp. $\gamma$ ) is a 123-avoiding sequence of elements in $[k+2, n]$ (resp. $[1, k-1])$ without successions, such that $\operatorname{red}(\lambda)=\gamma^{-1}$. 
Let $g(x)=\frac{-1+2 x+\sqrt{1-4 x^{2}}}{2 x-4 x^{2}}$ be the generating function for the set of involutions avoiding 123 (see [15]). Let $f(x)$ be the generating function of irreducible involutions avoiding 123. The above structure of $\sigma$ implies

$$
g(x)-f(x)=x^{4} \cdot h\left(x^{2}\right) \cdot g(x)+x^{2} \cdot h\left(x^{2}\right)
$$

where $h(x)$ is the generating function for irreducible permutations avoiding the pattern 123 (see Theorem 1). Finally, a simple calculation yields the desired results.

Theorem 17 For $\alpha \in\{132,213,321\}$, the generating function for the sets $\mathcal{I}_{n}^{\text {irr }}(\alpha)$, $n \geq 0$, of irreducible involutions of length $n$ avoiding $\alpha$ is given by (see [16], A125189):

$$
\frac{2(1+x)\left(1+x^{2}\right)}{1-x+x^{2}-x^{3}+(1+x) \sqrt{1-2 x^{2}-3 x^{4}}} .
$$

Proof: For $\alpha=132, \sigma \in \mathcal{I}_{n}^{i r r}(\alpha)$ can be written either $\sigma=\lambda n \beta \gamma k$ where $(\gamma k)^{-1}=\operatorname{red}(\lambda n) \in$ $\mathcal{S}_{k}^{i r r}(132)$ and such that $\beta \in \mathcal{I}_{n-2 k}^{\text {irr }}(132)$ or $\sigma=\beta n$ where $\beta \in \mathcal{I}_{n-1}^{\text {irr }}(\alpha)$ such that its last value is different from $n-1$. If $f(x)$ is the generating function for $\mathcal{I}_{n}^{\text {irr }}(\alpha)$, the above structure of $\sigma$ implies that $f(x)=g\left(x^{2}\right) f(x)+x g\left(x^{2}\right) f(x)+1+x$ where $g(x)$ is the generating function for irreducible permutations avoiding 132 and ending with $n$. So, we have $g(x)=x h(x)$ where $h(x)$ is the generating function for irreducible permutations avoiding 132 and not ending with $n$; thus $g(x)=x(M(x)-g(x)+1)$ where $M(x)=\frac{1-x-\sqrt{1-2 x-3 x^{2}}}{2 x}$ is the generating function for irreducible permutations avoiding 132 (see Theorem 2). A simple calculation gives the result for $\alpha=132$.

For $\alpha=213$, the result is obtained from $\alpha=132$ with the symmetry $\sigma \rightarrow\left(\sigma^{r}\right)^{c}$.

For $\alpha=321$, let $f(x)$ be the generating function for $\mathcal{I}_{n}^{\text {irr }}(\alpha)$. Then we have $f(x)=h(x)+g(x)$ where $h(x)$ (resp. $g(x)$ ) is the generating function for $\mathcal{D} \mathcal{I}_{n}^{\text {irr }}(\alpha)$ (resp. for $\mathcal{I}_{n}^{\text {irr }}(\alpha) \backslash \mathcal{D} \mathcal{I}_{n}^{\text {irr }}(\alpha)$ ). On the other hand, we have $f(x)=f_{1}(x)+f_{2}(x)$ and $g(x)=h(x) f_{1}(x)$ where $f_{1}(x)$ (resp. $f_{2}(x)$ ) is the generating function for permutations in $\mathcal{I}_{n}^{\text {irr }}(\alpha)$ with the last value equal to $n$ (resp. different from $n)$. Then we have $f_{1}(x)=x f_{2}(x), f_{1}(x)=\frac{x}{1+x} \cdot f(x)$ and $g(x)=\frac{x}{1+x} \cdot h(x) \cdot f(x)$. We obtain $f(x)=h(x)+\frac{x}{1+x} \cdot h(x) \cdot f(x)$. Since $h(x)$ is known using Theorem 12, a simple calculation gives the desired result.

Problem: It remains to obtain the generating function for the sets $\mathcal{I}_{n}^{\text {irr }}, n \geq 0$, of irreducible involutions of length $n$.

\begin{tabular}{|c|c|c|c|}
\hline Pattern & Sequence & Sloane & $a_{n}, n \geq 1$ \\
\hline\{\} & $?$ & $?$ & $1,3,5,13,37,107,341,1141$ \\
\hline$\{132\},\{213\},\{321\}$ & $\frac{2(1+x)\left(1+x^{2}\right)}{1-x+x^{2}-x^{3}+(1+x) \sqrt{1-2 x^{2}-3 x^{4}}}$ & $A 125189$ & $1,2,2,3,5,7,11,17,27,42$ \\
\hline$\{123\}$ & $\frac{1-2 x-2 x^{2}+4 x^{3}+\sqrt{1-4 x^{2}}}{(1-2 x)\left(1+2 x^{4}+\sqrt{1-4 x^{2}}\right)}$ & New & $1,3,4,9,16,31,58,112$ \\
\hline$\{231\},\{312\}$ & Tribonacci & $A 000213$ & $1,1,1,3,5,9,17,31,57$ \\
\hline
\end{tabular}

Tab. 4: Irreducible involutions avoiding at most one pattern of $\mathcal{S}_{3}$. 


\section{Acknowledgements}

I would like to thank the anonymous referees for their very careful reading of this paper and their helpful comments and suggestions.

\section{References}

[1] M.D. Atkinson and T. Stitt. Restricted permutations and the wreath product. Discrete Mathematics, 259(13):19-36, 2002.

[2] J.-L. Baril. Classical sequences revisited with permutations avoiding dotted pattern. Electr. J. Comb., 18(1), 2011.

[3] M. Bóna. Combinatorics of permutations. Chapman\&Hall, Chap. 4:129-173, 2004.

[4] E. Deutsch, A. Robertson, and D. Saracino. Refined restricted involutions. European Journal of Combinatorics, 28(1):481-498, 2007.

[5] R. Donaghey and L.W. Shapiro. Motzkin numbers. Journal of Combinatorial Theory, Series A, 23:291-301, 1977.

[6] I. Kaplansky. Symbolic solutions of certain problems in permutations. Bull. Amer. Math. Soc., 50:906-914, 1944.

[7] S. Kitaev and T. Mansour. A survey on certain pattern problems. University of Kentucky research report 200309. URL: http://www.ms.uky.edu/ math/MAreport/survey.ps.

[8] D.E. Knuth. The art of computer programming. Addison-Wesley, Reading, MA, Vol. 1, 1973.

[9] P. Manara and C. Perelli Cippo. The fine structure of 321 avoiding involutions. ArXiv:1010.5919v1, 2010.

[10] T. Mansour. Permutations avoiding a pattern from $S_{k}$ and at least two patterns from $S_{3}$. Ars. Combin., 62:227239, 2002.

[11] R. Parviainen. Wilf classification of bi-vincular permutation patterns. ArXiv:0910.5103v2, 2009.

[12] L. Pudwell. Enumeration schemes for permutations avoiding barred patterns. Electr. J. Comb., 17(1), 2010.

[13] J. Riordan. A recurrence for permutations without rising or falling successions. Ann. Math. Statist., 36:745-748, 1965.

[14] D.P. Roselle. Permutations by number of rises and successions. Proc. Amer. Math. Soc., 19:8-16, 1968.

[15] R. Simion and F.W. Schmidt. Restricted permutations. European Journal of Combinatorics, 6:383-406, 1985.

[16] N.J.A. Sloane. On-line encyclopedia of integer sequences. URL: http://www.research.att.com/ $\sim$ njas $/$ sequences/.

[17] R.P. Stanley. Catalan addendum, version of 22 october 2011. URL: http://www-math.mit.edu I rstan/ec/catadd.pdf.

[18] S.M. Tanny. Permutations and successions. Journal of Combinatorial Theory (A), 21:196-202, 1976.

[19] H. Tietze. Über gewisse unordnungen von permutationen. S.-B. Math.-Natur. Abt. Bayer. Akad. Wiss., pages 281-293, 1943.

[20] J. West. Permutations with forbidden subsequences and stack-sortable permutations. Ph.D. Thesis, MIT, 1990.

[21] J. West. Generating trees and forbidden subsequences. Proceeding of sixth FPSAC, pages 441-450, 1994.

[22] J. West. Generating trees and the catalan and schröder numbers. Discrete Math., 146:247-262, 1995. 